\section{Case Reports in Neurology}

Case Rep Neurol 2020;12:378-386

DOI: 10.1159/000510634

Published online: November 3, 2020 (c) 2020 The Author(s)

Published by S. Karger AG, Basel www.karger.com/crn

This article is licensed under the Creative Commons Attribution-NonCommercial 4.0 International License (CC BY-NC) (http://www.karger.com/Services/OpenAccessLicense). Usage and distribution for commercial purposes requires written permission.

\title{
A Patient with Limbic Encephalitis, Ear Perichondritis, and Episcleritis - An Unusual Presentation of Relapsing Polychondritis
}

\author{
Stanley Angkodjojo ${ }^{\mathrm{a}, \mathrm{b}} \quad$ Crystal Jing Jing $\mathrm{Yeo}^{\mathrm{c}-\mathrm{f}}$ \\ aDepartment of General Medicine (Rheumatology), Sengkang General Hospital, \\ Singapore, Singapore; bepartment of Rheumatology and Immunology, Singapore \\ General Hospital, Singapore, Singapore; 'Lee Kong Chian School of Medicine, \\ Singapore, Singapore; dExperimental Drug Development Center, Agency for Science, \\ Technology and Research (A*STAR), Singapore, Singapore; eInstitute of Molecular and \\ Cell Biology, Agency for Science, Technology and Research (A*STAR), \\ Singapore, Singapore; ${ }^{\mathrm{f}}$ National Neuroscience Institute, Singapore, Singapore
}

\section{Keywords}

Limbic encephalitis · Positron emission tomography/computed tomography · Relapsing polychondritis

\begin{abstract}
Relapsing polychondritis (RPC) is a rare autoimmune disease that is characterized by recurrent inflammation and destruction of cartilaginous tissues. Limbic encephalitis is a rare central nervous system manifestation of RPC that has been mentioned in case reports. Recognition of this association, and reliable methods of diagnosis, including the utility of neuroimaging modalities such as positron emission tomography/computed tomography (PET/CT) can be useful in the evaluation of this diagnostic challenge. We report a patient with limbic encephalitis associated with RPC, where PET/CT was effectively used in the diagnosis, and monitoring of response to
\end{abstract}




\section{Case Reports in Neurology}

Case Rep Neurol 2020;12:378-386

DOI: 10.1159/000510634

(c) 2020 The Author(s). Published by S. Karger AG, Basel www.karger.com/crn

Angkodjojo and Yeo: Limbic Encephalitis Associated with Relapsing Polychondritis

treatment. We also demonstrate that it can be a useful modality in certain situations when brain magnetic resonance imaging cannot be done.

\section{Introduction}

Relapsing polychondritis (RPC) is a rare disease that occurs among individuals of all races and age groups. It is an immune-mediated disorder that is characterized by recurrent and progressive inflammation of cartilaginous structures and other tissues throughout the body, particularly the ears, nose, eyes, joints, and respiratory tracts [1]. In unusual cases, RPC presents with primarily neurological manifestations [2]. Rare neurological complications associated with RPC include hemiplegia, seizures, metabolic encephalopathy, dementia, myelitis, peripheral neuropathy, aseptic meningitis, lymphocytic meningoencephalitis, rhomboencephalitis, limbic encephalitis, and pachymeningitis related to polyangiitis with granulomatosis [2-4].

Herein, we describe the case of an unusual presentation of RPC in a patient with nonherpetic non-paraneoplastic limbic encephalitis, ear perichondritis, and episcleritis. We also illustrate the role of positron emission tomography/computed tomography (PET/CT) as an effective tool for the diagnosis and assessment of response to therapy in our patient with limbic encephalitis.

\section{Case Presentation}

A 66-year-old Chinese Singaporean man was admitted to our hospital for subacute mental confusion, forgetfulness of recent events, disorientation and getting lost, hyperactive behaviour, and emotional lability progressing over 6 weeks. His family members noted that he was becoming increasingly restless and agitated and could no longer keep up with the demands of his job as a regional sales manager in a merchandising company. He had lost $7 \mathrm{~kg}$ over the 6 weeks preceding his admission, with no other constitutional symptoms such as fever and night sweats. 2 months prior to admission, he developed pain, swelling and erythema of his right ear which improved slightly with over-the-counter nonsteroidal anti-inflammatory drugs. He also had a history of bilateral episcleritis successfully treated with steroid eye drops prescribed by an ophthalmologist 6 months before his current presentation. Other past medical history was significant for well-controlled diabetes mellitus on diet restriction and metformin (glycated haemoglobin, $\mathrm{HbA}_{1 \mathrm{c}}$, was 6.4-6.7\%), and ischaemic cardiomyopathy for which he underwent implantable cardioverter defibrillator (ICD) implantation in 2012.

On admission, his body temperature was $36.7^{\circ} \mathrm{C}$, blood pressure was $112 / 66 \mathrm{~mm} \mathrm{Hg}$ and heart rate was 88 beats per minute. Physical examination revealed a floppy-eared appearance of his right ear. He was confabulating and disoriented to time, person and place, and had a limited recall of recent events. He was unable to do serial 7s and had difficulties with shortterm recall. He scored a markedly low score of 11/30 on the Mini-Mental States Examination (MMSE). Coordination was moderately impaired, with difficulties with tandem gait and pastpointing on finger to nose testing. Cranial nerves were normal. Tone, strength and reflexes were normal throughout. Sensory examination was normal. The rest of the examination of his eyes, nose, cardiovascular, respiratory, and abdominal systems were unremarkable. There were no palpable lymph nodes, goitre, rash, joint swelling, alopecia, mouth ulcers, or other features of connective tissue diseases (CTD). 


\section{Case Reports in Neurology}

Case Rep Neurol 2020;12:378-386

DOI: 10.1159/000510634

(c) 2020 The Author(s). Published by S. Karger AG, Basel www.karger.com/crn

Angkodjojo and Yeo: Limbic Encephalitis Associated with Relapsing Polychondritis

Laboratory studies revealed an inflammatory reaction that included raised white blood cell count of $15.41 \times 10^{9} / \mathrm{L}$ (normal 4.0-10.0 $\times 10^{9} / \mathrm{L}$ ), increased erythrocyte sedimentation rate of $83 \mathrm{~mm} / \mathrm{h}$ and C-reactive protein of $72.8 \mathrm{mg} / \mathrm{L}$ (normal $0.2-9.1 \mathrm{mg} / \mathrm{L}$ ). Blood tests and investigations were negative for factors that might cause altered mental status and impaired consciousness, such as electrolyte imbalances, uraemia, thyroid dysfunction, liver dysfunction, vitamin $B_{12}$ deficiency and abnormal urine and serum drug toxicology. Syphilis, herpes simplex virus (HSV)-1, HSV-2, varicella zoster virus (VZV), human herpes virus 6 (HHV-6), cytomegalovirus (CMV), Epstein-Barr virus (EBV), human immunodeficiency virus (HIV), measles, rubella, and mumps were serologically excluded. Blood and urine cultures for bacteria were also negative. A lumbar puncture was performed and cerebrospinal fluid (CSF) showed a raised protein concentration of $>2.00 \mathrm{~g} / \mathrm{L}$ (normal $0.1-0.4 \mathrm{~g} / \mathrm{L}$ ), normal glucose of $3.6 \mathrm{mmol} / \mathrm{L}$ (serum glucose, $5.5 \mathrm{mmol} / \mathrm{L}$ ), mild pleocytosis of white cells 48 cells $/ \mu \mathrm{L}$ (74\% lymphocytes), and cytology yielding no malignant cells. Computed tomography (CT) with a follow-up angiogram of the brain showed no evidence of territorial infarcts, intracranial haemorrhage, or vessel wall alterations to suggest cerebral vasculitis. Continuous electroencephalogram (EEG) showed diffuse slowing of background activities to $1-2 \mathrm{~Hz}$, with no epileptic focus or delta brush. Our patient was commenced on empiric treatment for infectious meningoencephalitis, which included anti-bacterial, anti-viral, and treatment for Mycobacterium tuberculosis, without improvement. By day 4 of admission, he had become catatonic, mute and akinetic with generalized rigidity and dystonia in the limbs. At this point, CSF microbiological studies for neurotrophic viruses (HSV, VZV, CMV, enterovirus), bacteria, Mycobacterium tuberculosis, and fungal organisms returned negative. MRI of the brain could not be done due to the presence of an MRI-incompatible ICD. Due to suspicion for paraneoplastic or autoimmune limbic encephalitis in view of his symptoms, age and unintentional weight loss, whole-body PET/CT scan with ${ }^{18} \mathrm{~F}$-fluorodeoxyglucose (FDG) was done instead which revealed a focus of intense metabolic activity in the left hippocampal region (Fig. 1a-c) and right ear skin thickening with corresponding increased metabolic activity (Fig. 1d, e). There was no evidence for occult malignancy and systemic vasculitis. Autoimmune workup for CTDs including rheumatoid factor, antinuclear antibodies (ANA), anti-double stranded DNA (anti-dsDNA), antibodies against extractable nuclear antigens, antineutrophil cytoplasmic antibodies (ANCA), anti-thyroid peroxidase (anti-TPO) antibodies was negative. Serum and CSF paraneoplastic/autoimmune encephalitis auto-antibodies including voltage-gated potassium channel complex, anti-neuronal antibodies, NMDAR, CASPR2, LGI1, AMPARR1/2, DPPX and GABA $A_{B} R$ were negative.

Subsequent biopsy of his right ear revealed chronic infiltration of inflammatory cells in the perichondrium (Fig. 2). A diagnosis of RPC was made based on the presence of ocular inflammation (given his history of episcleritis), and biopsy confirmation of auricular cartilage inflammation. Considering his clinical symptoms of subacute cognitive impairment and behavioural changes, signal changes within limbic structures on PET/CT, and lymphocytosis and elevated protein levels on CSF analysis, limbic encephalitis was also diagnosed. As causes of encephalopathy including metabolic disorders, drugs and toxins, infectious causes such as HSV and HIV, tumour-associated limbic encephalitis, Hashimoto's encephalopathy, and other CTDs such as systemic lupus erythematosus (SLE), Behçet's disease and Sjögren's syndrome were ruled out, limbic encephalitis associated with RPC was diagnosed.

Our patient was commenced on intravenous methylprednisolone pulse therapy $(1,000$ $\mathrm{mg}$ per day for 5 days) followed by oral prednisolone (60 $\mathrm{mg}$ per day). He also received 3 cycles of monthly intravenous cyclophosphamide. His cognitive function, emotional lability and problematic behaviour improved gradually over the ensuing 3 months post-discharge. 


\section{Case Reports in Neurology}

Case Rep Neurol 2020;12:378-386

DOI: $10.1159 / 000510634$

(c) 2020 The Author(s). Published by S. Karger AG, Basel www.karger.com/crn

Angkodjojo and Yeo: Limbic Encephalitis Associated with Relapsing Polychondritis

His coordination difficulties completely resolved, and he could continue active daily living independently with minimal supervision, though he could not resume his work. MMSE score also recovered to 24/30 after a 3-month period. Oral prednisolone was tapered, and he remained stable on low-dose prednisolone. A follow-up PET/CT was done 3 months after his initial presentation and it showed interval resolution of the increased FDG uptake within the left hippocampus (Fig. 3) and right external ear.

\section{Discussion}

RPC is a rare disease that is characterized by recurrent and progressive inflammation of cartilaginous structures and other tissues throughout the body. The annual incidence has been estimated to range up to 3.5 per million persons from population studies [5]. According to McAdam et al. [1], a definite diagnosis of RPC is made when 3 of the following 6 clinical features, including auricular and nasal chondritis, nonerosive inflammatory polyarthritis, ocular inflammation, respiratory tract chondritis, and audio-vestibular dysfunction, are present along with a histologically compatible biopsy (ear, nose, respiratory tract). Due to the variability of clinical manifestations occurring at a given point in time, Damiani and Levine [6] subsequently proposed a modification to the McAdam criteria which is now commonly used to confirm the diagnosis of RPC: (a) at least 3 of McAdam's diagnostic criteria; (b) 1 or more of McAdam's clinical criteria with biopsy confirmation of cartilage inflammation; or (c) chondritis at 2 or more separate anatomic locations with response to glucocorticoids and/or dapsone. Our case fits criterion (b).

Neurological involvement in RPC is even rarer and only occurs in 3\% of patients [2]. Diagnosis of neurological complications associated with RPC is often challenging due to its rarity and heterogenous manifestations [2-4]. Our patient presented with limbic encephalitis during the course of RPC, a rare occurrence which has only been described in a few case reports to date [4]. Stewart et al. [7] reported extensive cerebral and systemic vasculitis to be the cause of central nervous system involvement in RPC. Multifocal neurological abnormalities and lesions on neuroimaging supports the hypothesis of vasculitis as the etiopathology behind central nervous system manifestations in RPC [7]. In contrast, other authors have reported inflammatory changes non-specific to vasculitis in patients presenting with limbic encephalitis associated with RPC [4]. Like other forms of limbic encephalitis, these are likely to be antibody mediated as anti-cartilage antibodies have been detected in RPC patients and correlate with disease severity, and glutamate receptor and glycospingolipid antibodies have been detected in CSF and serum of RPC patients with limbic encephalitis [8]. Our patient's neurological symptoms and PET/CT findings of a unilateral left hippocampal lesion with no apparent multifocal lesion are similar to those of patients with non-herpetic limbic encephalitis rather than vasculitis [4]. Viral aetiologies and seizures which might cause a unilateral mesial temporal lesion were excluded by serologies and EEG monitoring.

The clinical features of limbic encephalitis are diverse and early diagnosis of the disorder is frequently difficult. Graus et al. [9] proposed a consensus criteria that definite diagnosis of limbic encephalitis can be made when all 4 of the following criteria have been met: (1) subacute onset (less than 3 months) of cognitive deficits, seizures, or psychiatric symptoms, (2) bilateral brain abnormalities in medial temporal lobes in T2-weighted MRI images, (3) CSF pleocytosis ( $>5$ cells per $\mathrm{mm}^{3}$ ) or EEG with epileptic discharges or slow-wave activity involving the temporal lobes, and (4) reasonable exclusion of alternative causes such as infectious, inflammatory, metabolic, neurovascular and neurodegenerative aetiologies. 


\section{Case Reports in Neurology}

Case Rep Neurol 2020;12:378-386

DOI: $10.1159 / 000510634$

(c) 2020 The Author(s). Published by S. Karger AG, Basel www.karger.com/crn

Angkodjojo and Yeo: Limbic Encephalitis Associated with Relapsing Polychondritis

Determination of the pathogenic antibody can help to give a more accurate diagnosis and prognosis as intra-neuronal antibodies against $\mathrm{Hu}$, Ma and $\mathrm{Ri}$ are usually associated with an occult neoplasm and less responsive to immunotherapy, whereas cell-surface antibodies against VGKC, AMPA and GAD antibodies are more responsive to immunotherapy and have a better prognosis. It has been noted that around $7-26 \%$ of all autoimmune limbic encephalitis cases present without detectable antibodies [10]. It is imperative to note that absence of antibodies does not necessarily exclude an autoimmune encephalitis, as in our case, and should not delay treatment as early immunotherapy improves outcome.

Many patients with a diagnosis of limbic encephalitis may have normal or non-specific MRI findings. In these circumstances, PET/CT can be key in clinching the diagnosis of limbic encephalitis, such as in our patient. In the absence of FLAIR/T2 abnormalities of the medial temporal lobes on MRI, FDG-PET hypermetabolism in the medial temporal lobes may meet this requirement for the autoimmune encephalitis consensus criteria [9]. Indeed, PET/CT has been reported to be more often abnormal compared to initial CSF studies, MRI, or EEG in neurology inpatients with limbic encephalitis and is the earliest abnormal sign [11]. Whilst brain MRI is more commonly performed in clinical practice due to reasons such as greater accessibility, experience of requesting physicians, and lower cost, the addition of FDG-PET/CT seems to be a reasonable way to enhance the sensitivity of the diagnostic algorithm, especially in patients with normal MRI scans [12]. PET/CT can reveal abnormalities in more areas of the brain, making it a more sensitive test (78\%) compared to MRI (63\%) to aid the often-challenging diagnosis of limbic encephalitis [12]. PET/CT can also be useful in situations where MRI is contraindicated, such as the presence of MRI-incompatible ICD, cardiac pacemaker, and allergy to MRI contrast agents. Our patient did have subacute onset of cognitive deficits and psychiatric symptoms, CSF pleocytosis, EEG slowing, PET/CT abnormalities, and reasonable exclusion of alternative causes, consistent with the consensus criteria for autoimmune limbic encephalitis.

This case also suggests that PET/CT may aid in the management of limbic encephalitis by correlating disease activity in the brain to clinical response to treatment. Previous case studies demonstrated that the recovery of clinical symptoms is coincident with normalization of the pattern of cerebral glucose metabolism [13]. Congruent with this, PET/CT in our patient showed resolution of the hypermetabolism of the left temporal lobe 3 months after immunotherapy, which correlated with his good clinical response to treatment. Although standard first-line therapy with steroids could at least theoretically affect results by increasing blood glucose levels, nonetheless, this condition is likely to cause a global and homogeneous reduction in FDG uptake, and the foci of hyper- or hypometabolism corresponding to the neurologic lesions can be detected with a high degree of certainty [14]. In addition, our case also illustrates that whole-body PET/CT, in addition to looking for occult neoplasm, can help assess systemic inflammatory disease activity, as seen from the interval resolution of hyper-metabolic activity correlating with perichondritis in his right ear with no new inflammatory lesions. This suggested that the primary autoimmune condition underlying the limbic encephalitis, RPC, was satisfactorily treated. Lastly, whole-body PET/CT may be a more sensitive and cost-effective strategy to look for occult neoplasm, as compared to performing brain MRI with complementary tests for cancer screening (whole-body CT scans, gynaecologic ultrasound scans, mammograms, and gastrointestinal endoscopies), in the evaluation process of a patient with suspected autoimmune encephalitis [15]. 


\section{Case Reports in Neurology}

\begin{tabular}{l|l}
\hline Case Rep Neurol 2020;12:378-386 \\
\hline DOI: 10.1159/000510634 & $\begin{array}{l}\text { (c) 2020 The Author(s). Published by S. Karger AG, Basel } \\
\text { www.karger.com/crn }\end{array}$ \\
\hline
\end{tabular}

Angkodjojo and Yeo: Limbic Encephalitis Associated with Relapsing Polychondritis

\section{Conclusion}

Although RPC is a rare disorder, it should be considered in the differential diagnosis of neurological conditions such as limbic encephalitis. It is also worth noting that there are many benefits of ${ }^{18} \mathrm{~F}-\mathrm{FDG}$ PET/CT imaging in the management of patients with limbic encephalitis. PET/CT should be integrated with other clinical or imaging investigations in the diagnostic algorithm and monitoring of such patients. While further studies are warranted to validate other less investigated indications of PET/CT in limbic encephalitis, such as the evaluation of response to treatment, we have shown that it is a useful modality in certain situations when brain MRI cannot be done.

\section{Acknowledgements}

We thank Dr. Timothy Tay Kwang Yong, Consultant, from Department of Anatomical Pathology, Singapore General Hospital, for the histopathological images of the patient's right ear biopsy. We also thank Dr. Khor Yiu Ming, Consultant, from Department of Nuclear Medicine and Molecular Imaging, Singapore General Hospital, for the FDG PET/CT images.

\section{Statement of Ethics}

There are no ethical conflicts to declare. The patient was informed of the purpose of the case presentation, and provided written informed consent for the publication, including images, case history, and clinical data.

\section{Disclosure Statement}

The authors have no conflicts of interest to declare.

\section{Funding Sources}

The authors have no funding sources to declare.

\section{Author Contributions}

All authors read and approved the manuscript before submission. All authors contributed equally to the review of the clinical data, literature search, and drafting of the manuscript.

\section{References}

1 McAdam LP, O'Hanlan MA, Bluestone R, Pearson CM. Relapsing polychondritis: prospective study of 23 patients and a review of the literature. Medicine (Baltimore). 1976 May;55(3):193-215.

2 Hatti K, Giuliano V. Central nervous system involvement in relapsing polychondritis. J Clin Rheumatol. 2014 Oct;20(7):396-7. 


\section{Case Reports in Neurology}

3 Cañas CA, Díaz-Martínez JC, Tobón GJ. Combination of hypertrophic pachymeningitis, PR3-ANCA-positive vasculitis, and relapsing polychondritis. J Rheumatol. 2011 May;38(5):966-7.

4 Fujiki F, Tsuboi Y, Hashimoto K, Nakajima M, Yamada T. Non-herpetic limbic encephalitis associated with relapsing polychondritis. J Neurol Neurosurg Psychiatry. 2004 Nov;75(11):1646-7.

5 Kent PD, Michet CJ Jr, Luthra HS. Relapsing polychondritis. Curr Opin Rheumatol. 2004 Jan;16(1):56-61.

6 Damiani JM, Levine HL. Relapsing polychondritis-report of ten cases. Laryngoscope. 1979 Jun;89(6 Pt 1):929-46.

7 Stewart SS, Ashizawa T, Dudley AW Jr, Goldberg JW, Lidsky MD. Cerebral vasculitis in relapsing polychondritis. Neurology. 1988 Jan;38(1):150-2.

8 Kashihara K, Kawada S, Takahashi Y. Autoantibodies to glutamate receptor GluRepsilon2 in a patient with limbic encephalitis associated with relapsing polychondritis. J Neurol Sci. 2009 Dec;287(1-2):275-7.

9 Graus F, Titulaer MJ, Balu R, Benseler S, Bien CG, Cellucci T, et al. A clinical approach to diagnosis of autoimmune encephalitis. Lancet Neurol. 2016 Apr;15(4):391-404.

10 Graus F, Escudero D, Oleaga L, Bruna J, Villarejo-Galende A, Ballabriga J, et al. Syndrome and outcome of antibody-negative limbic encephalitis. Eur J Neurol. 2018 Aug;25(8):1011-6.

11 Probasco JC, Solnes L, Nalluri A, Cohen J, Jones KM, Zan E, et al. Abnormal brain metabolism on FDG-PET/CT is a common early finding in autoimmune encephalitis. Neurol Neuroimmunol Neuroinflamm. 2017 May;4(4):e352.

12 Baumgartner A, Rauer S, Mader I, Meyer PT. Cerebral FDG-PET and MRI findings in autoimmune limbic encephalitis: correlation with autoantibody types. J Neurol. 2013 Nov;260(11):2744-53.

13 Leypoldt F, Buchert R, Kleiter I, Marienhagen J, Gelderblom M, Magnus T, et al. Fluorodeoxyglucose positron emission tomography in anti-N-methyl-D-aspartate receptor encephalitis: distinct pattern of disease. J Neurol Neurosurg Psychiatry. 2012 Jul;83(7):681-6.

14 Quartuccio N, Caobelli F, Evangelista L, Alongi P, Kirienko M, De Biasi V, et al.; Young AIMN Working Group. The role of PET/CT in the evaluation of patients affected by limbic encephalitis: A systematic review of the literature. J Neuroimmunol. 2015 Jul;284:44-8.

15 McKeon A, Apiwattanakul M, Lachance DH, Lennon VA, Mandrekar JN, Boeve BF, et al. Positron emission tomography-computed tomography in paraneoplastic neurologic disorders: systematic analysis and review. Arch Neurol. 2010 Mar;67(3):322-9. 


\section{Case Reports in Neurology}
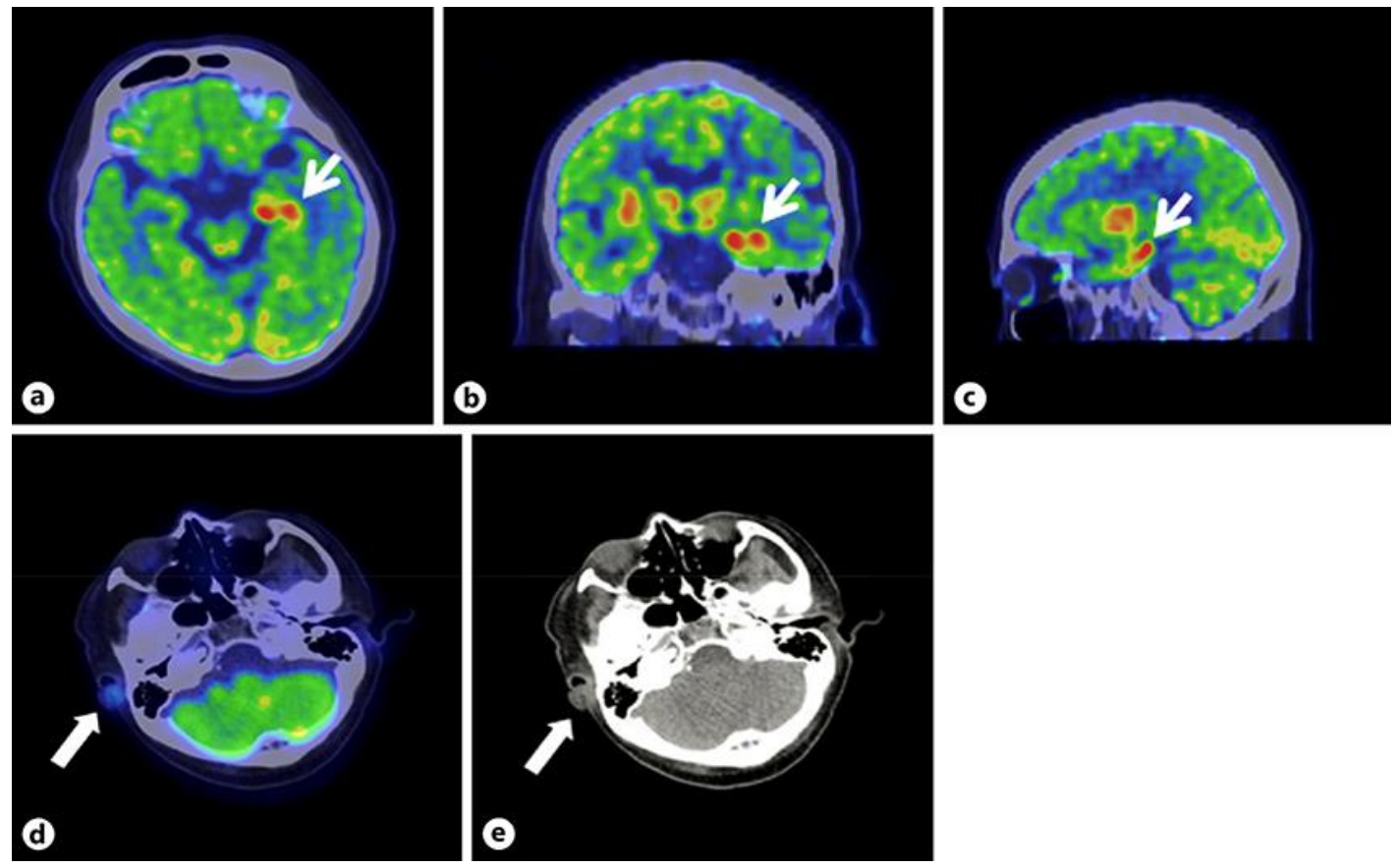

Fig. 1. Initial FDG PET/CT scan shows a focus of intense metabolic activity in the left hippocampus (white arrow) above that of the background metabolic activity in the rest of the brain parenchyma in axial (a), coronal (b) and sagittal (c) views. Fused FDG PET/CT shows moderately increased metabolic activity at the right ear (d) corresponding to mild skin thickening on the CT (e).
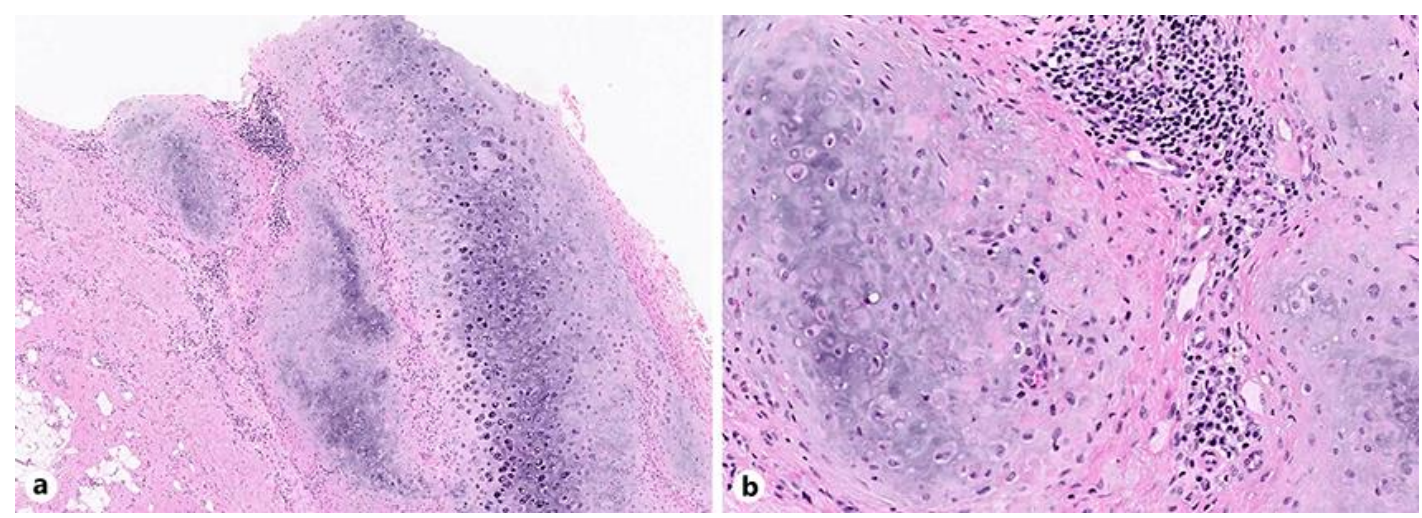

Fig. 2. Histopathological examination of right ear biopsy (haematoxylin-eosin [HE] stain) showed infiltration of pinna cartilage with inflammatory cells (predominantly lymphocytes with few plasma cells) in the perichondrium. a Low magnification view (HE stain $\times 5$ ). $\mathbf{b}$ Higher magnification view (HE stain $\times 20$ ). 
Case Reports in Neurology
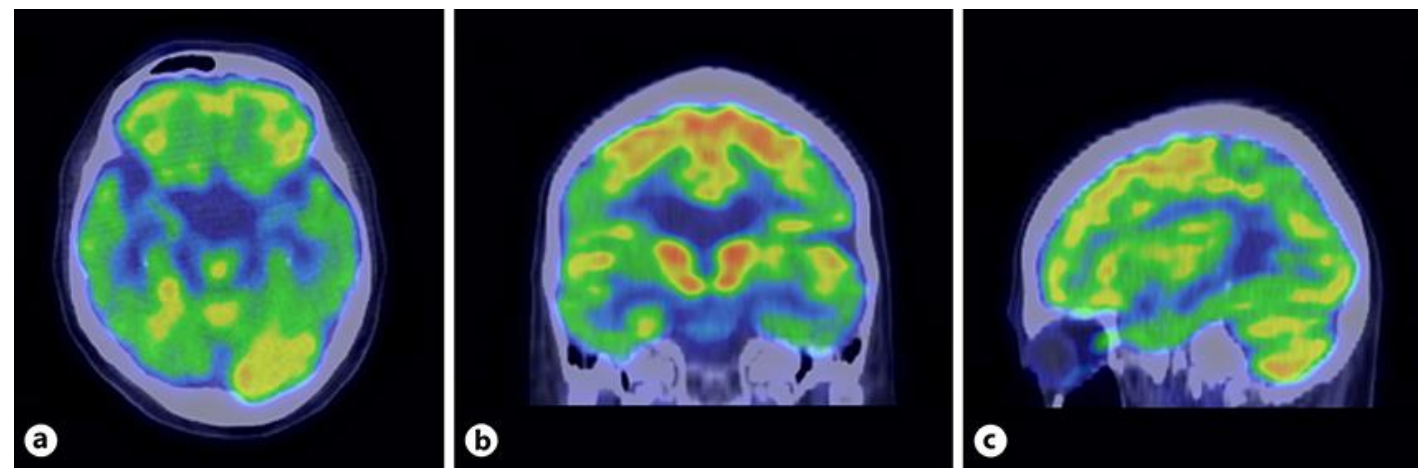

Fig. 3. FDG PET/CT scan performed 3 months after initial presentation showed interval resolution of the focus of intense metabolic activity in axial (a), coronal (b) and sagittal (c) views. 